\title{
Simplified Ground-type Single-plate Electrowetting Device for Droplet Transport
}

\author{
Jong-Hyeon Chang*, Dong-Sik Kim** and James Jungho Pak ${ }^{\dagger}$
}

\begin{abstract}
The current paper describes a simpler ground-type, single-plate electrowetting configuration for droplet transport in digital microfluidics without performance degradation. The simplified fabrication process is achieved with two photolithography steps. The first step simultaneously patterns both a control electrode array and a reference electrode on a substrate. The second step patterns a dielectric layer at the top to expose the reference electrode for grounding the liquid droplet. In the experiment, a $5 \mu \mathrm{m}$ thick photo-imageable polyimide, with a 3.3 dielectric constant, is used as the dielectric layer. A $10 \mathrm{~nm}$ Teflon-AF is coated to obtain a hydrophobic surface with a high water advancing angle of $116^{\circ}$ and a small contact angle hysteresis of $5^{\circ}$. The droplet movement of $1 \mathrm{mM}$ methylene blue on this simplified device is successfully demonstrated at control voltages above the required $45 \mathrm{~V}$ to overcome the contact angle hysteresis.
\end{abstract}

Keywords: Electrowetting, Digital microfluidics, Droplet transport, Single-plate configuration

\section{Introduction}

Electrowetting is a microfluidic phenomenon, which induces the apparent contact angle change of a conductive liquid droplet with an applied electric field. Applying voltage between the conductive liquid and the electrode beneath the dielectric layer can vary the liquid contact angle according to the following Lippmann-Young equation:

$$
\cos \theta_{V}=\cos \theta_{0}+\frac{c V^{2}}{2 \gamma_{l v}}
$$

where $\theta_{0}, \theta_{V}, c, \gamma_{l v}$, and $V$ are the inherent contact angle, the changed contact angle by electrowetting, the capacitance per unit area of the dielectric layer (in $\mathrm{F} / \mathrm{m}^{2}$ ), the liquidvapor interfacial tension (in $\mathrm{N} / \mathrm{m}$ or $\mathrm{J} / \mathrm{m}^{2}$ ) and the applied voltage across the dielectric (in V), respectively [Fig. 1(a)]. The lateral motion of the droplet can be achieved when the electrowetting effect is partially generated at the threephase contact line of the liquid droplet on the activated electrode of the arrayed control electrodes. The electrowetted advancing angle $\left(\theta_{V, a}\right)$ is smaller than the inherent receding angle $\left(\theta_{0, r}\right)$ [Fig. 1(b)]. The electrowetting system has advantages of low power consumption and fast response. Thus, it has wide applications, not only in digital microfluidics, but also in liquid lenses and displays [1-4].

$\dagger$ Corresponding author: School of Electrical Engineering, Korea University, Korea. (pak@korea.ac.kr)

* Semiconductor Research Institute, Korea University, Korea. (ican@korea.ac.kr)

** Korea University College of Medicine, Korea. (kimds1@korea.ac.kr)

Received: November 19, 2010; Accepted: March 31 10, 2011

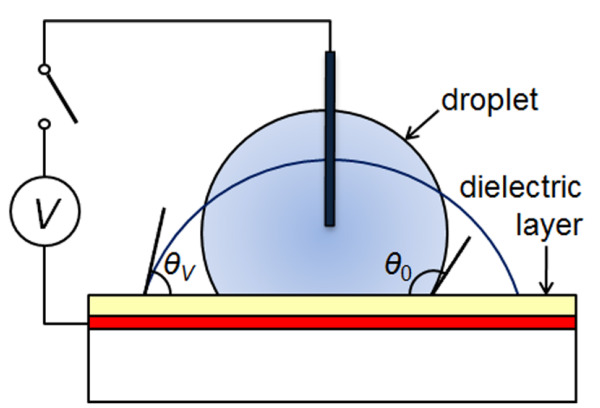

(a)

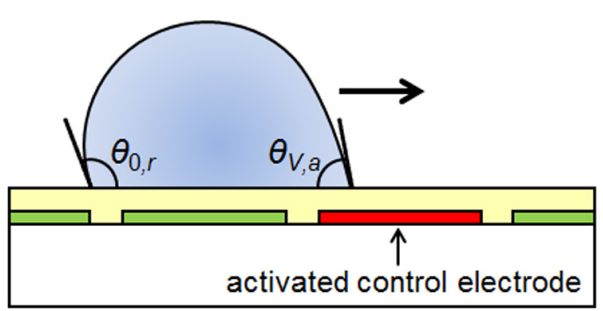

(b)

Fig. 1. Schematic cross-sections of (a) contact angle change by electrowetting on dielectric, and (b) electrowetting droplet transport on dielectric along the patterned electrode array.

Electrowetting in digital microfluidics are generally divided into two-plate and single-plate configurations. A typical two-plate configuration has a control electrode array, a dielectric layer on the bottom plate, and a reference electrode on the top plate [5]. On the other hand, in a single-plate configuration, both control and reference electrodes are on the bottom plate. Droplets can be manipulated without the top plate. More complex microfluidic 
operations are possible only in two-plate devices. Such operations include droplet splitting or dispensing for lab on a chip application. However, single-plate devices have some advantages, such as faster mixing, larger droplet volume manipulation on a given footprint, and easier access to droplets for handling or optical detection [6]. The top plate can be utilized for another purpose if the top plate in the two-plate devices is freed from the electrowetting actuation and the process can be performed using only the bottom plate as a single-plate device. This can serve as controllable heater electrodes, magnetic components, sensors, and specific chemistry or structures appropriate for each application $[7,8]$.

Single-plate electrowetting configurations are also divided into ground-type and unground-type [9]. Previously reported ground-type devices allow lower voltage electrowetting. However, their fabrication process is more complex compared with that of the unground-type devices. The current paper presents a simplified and more efficient way of fabricating a ground-type single-plate electrowetting device. This device can also operate without any degradation compared with previously reported devices.

\section{Design and Fabrication}

In 1998, Washizu first reported on electrostatic actuation of liquid droplets on the unground-type single-plate device [10]. Torkkeli et al. then improved the actuation with superhydrophobic surfaces [11]. The patterned serial line electrodes underneath a dielectric layer were activated one by one for droplet transport [Fig. 2(a)]. Yi and Kim have reported a parallel electrode design for electrowetting droplet transport [Fig. 2(b)] [12]. Moon and Kim have shown asymmetric electrowetting characteristics that can also generate droplet transport when using a series of square electrodes [Fig. 2(c)] [13, 14]. The advantage of an unground-type electrowetting device is its simple fabrication process. However, its electrowetting force is lower compared with that of the ground-type electrowetting device. The horizontal electrowetting force $\left(F_{e x}\right)$ acting on the droplet is generated along the effective three-phase contact line, as shown in the following equation [15]:

$$
F_{e x}=\frac{1}{2} c V^{2} \text {. }
$$

The lower force is attributed to the fact that only a portion of the applied voltage contributes to the electrowetting force generation. The applied voltage is dropped across two dielectric layers. One part is between the activated control electrode and the droplet, and the other part is between the reference electrode and the droplet. The electrowetting force is proportional to the square of the applied voltage across the dielectric layer. Thus, the electrowetting force is further reduced when the voltage drop across the dielectric layer decreases. Fouillet and Achard have reported a ground-type single-plate device with floating electric wires for grounding droplets [Fig. 2(d)] [16]. However, these electric wires make the fabrication process quite complicated. Therefore, the grounding line electrode was patterned on the dielectric layer [Figs. 2(e)-(f)] [17, 18].
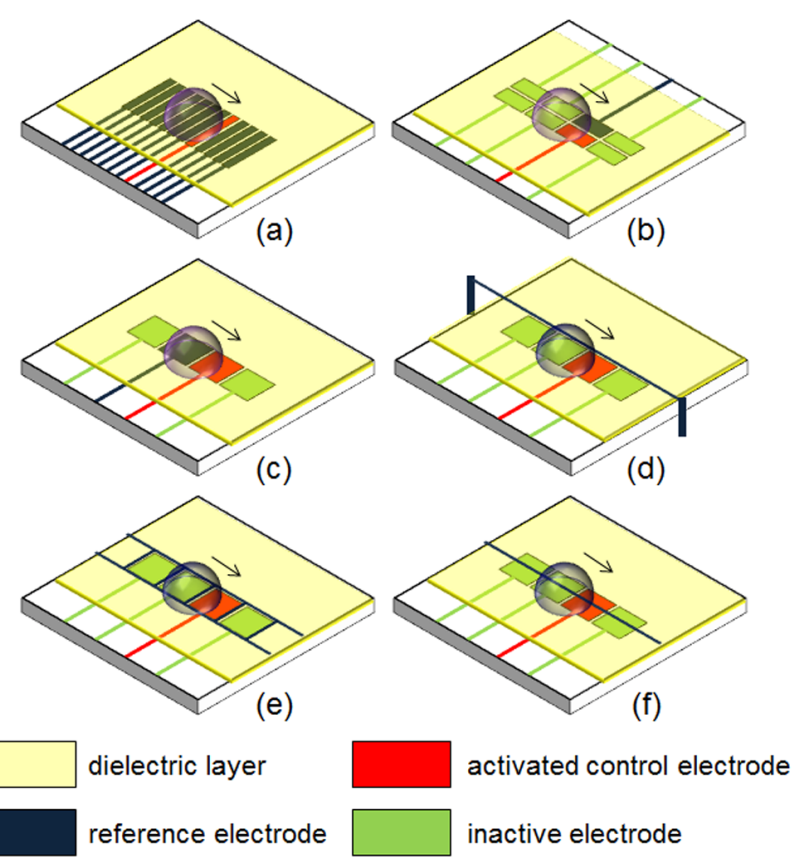

Fig. 2. Schematics of various single-plate electrowetting configurations for droplet transport: (a)-(c) Unground-type; (d)-(f) Ground-type.

As mentioned above, the ground-type single-plate configurations are more efficient in terms of electrowetting performance than the unground-type. However, they require an additional fabrication step to pattern the top reference electrode on the dielectric layer. Their fabrication process generally requires three photolithography steps: bottom electrode patterning on a substrate for the control electrode array; insulating dielectric layer patterning; and top reference electrode patterning. These steps can be further simplified by patterning both the control electrode array and the reference electrode in one step after depositing the electrode metal on the substrate. Afterwards, an insulating dielectric layer can be deposited on the whole surface and patterned to simultaneously expose both the reference electrode and the connection pads. This results in the proposed simplified process. The droplet cannot be grounded unless the reference electrode is exposed. The voltage drop occurs through two dielectric layers, namely, on the activated control and reference electrodes. The electrowetting force then becomes weaker than when the droplet is grounded. Therefore, the reference electrode was designed for exposure not only for fabrication efficiency, but also for driving efficiency. Fig. 3 shows a schematic view of the described coplanar ground-type electrowetting de- 
vice and its cross-sectional view along A-A' after each major fabrication step.

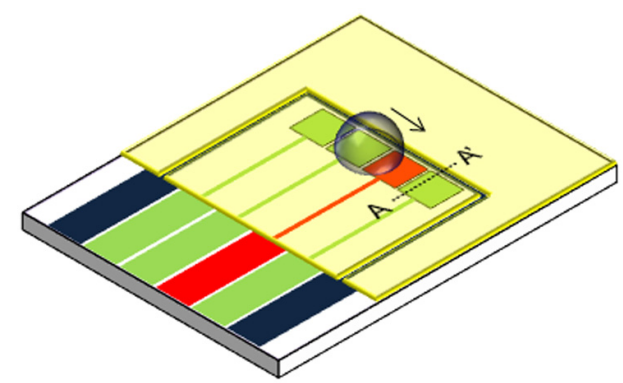

(a)

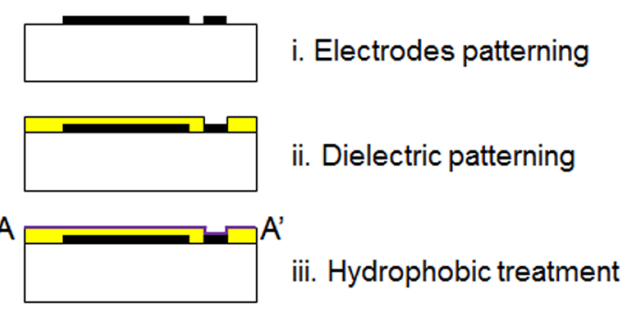

(b)

Fig. 3. A schematic view of the coplanar ground-type electrowetting device and its fabrication process: (a) 3dimensional view of the device; (b) Cross-sectional view along $A-A^{\prime}$ in (a) after major fabrication process steps.

For both reference electrode and control electrode array of the proposed devices, $100 \mathrm{~nm} \mathrm{Cr}$ was deposited on a glass substrate by electron beam evaporation. These were simultaneously patterned by wet etching with a photoresist (AZ1512, Clariant) mask. The control electrodes consisted of an array of $1.2 \times 1.2 \mathrm{~mm}$ squares with $50 \mu \mathrm{m}$ space. The reference electrode was single line next to the control electrode array with a width of a $200 \mu \mathrm{m}$. Polyimide (Durimide 7510, Fujifilm) was spin-coated at $5000 \mathrm{rpm}$ for $30 \mathrm{~s}$ and then patterned by photolithography for opening the reference electrode and connection pads. This photo-imageable polyimide was used as a dielectric layer to make the fabrication process simpler. Unless the dielectric material can be directly patterned, a more complex process for patterning the dielectric layer would be required. This may include performing photolithography with an additional photoresist, dry or wet etching the dielectric layer, and removing the photoresist.

After hard-baking at $350{ }^{\circ} \mathrm{C}$ for $1 \mathrm{~h}$ in $\mathrm{N}_{2}$, the resulting polyimide thickness was $5 \mu \mathrm{m}$. Durimide 7500 series polyimides are photo-imageable and are known to have a 3.3 dielectric constant and a $345 \mathrm{~V} / \mu \mathrm{m}$ dielectric strength. For curing polyimide, its residual solvent was removed in the hard-bake process, after which the desired surface properties were finalized. When the patterned polyimide was hard-baked in atmospheric environment, the cured film color changed to dark brown [Fig. 4(a)] possibly due to oxidation of the residual solvent in the film. Only after hard-baking in an inert gas, such as $\mathrm{N}_{2}$, can highly transparent polyimide film be obtained [Fig. 4(b)].

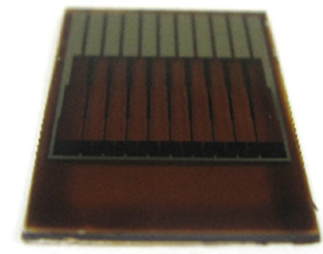

(a)

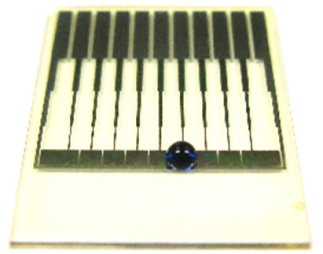

(b)
Fig. 4. Optical images of the fabricated ground-type singleplate electrowetting devices using polyimide dielectric cured in (a) atmospheric and (b) $\mathrm{N}_{2}$ environment.

Finally, $0.2 \mathrm{wt} \%$ Teflon-AF (601S2-100-6, DuPont) diluted in a fully-fluorinated solvent (FC-40, 3M) was spincoated at $1500 \mathrm{rpm}$ for $30 \mathrm{~s}$ and baked at $200{ }^{\circ} \mathrm{C}$ for $30 \mathrm{~min}$. This thin Teflon-AF layer was used to make the whole surface hydrophobic in order to enlarge the contact angle variation on electrowetting devices without electrically insulating the reference electrode from the liquid droplet [5]. The resulting Teflon-AF thickness measured by an ellipsometer (FE-VIID, Rudolph) was $\sim 10 \mathrm{~nm}$. The water advancing contact angle was $116^{\circ}$ with the contact angle hysteresis of $5^{\circ}$. These were measured by a contact angle analyzer (Phoenix 300, Surface Electro Optics).

\section{Results and Discussion}

The fabricated chip was connected to a DC power supply (2400 SourceMeter, Keithley) with a peripheral component interconnect slot to apply voltage between one of the control electrodes and the reference electrode in contact with a droplet. A droplet of $2.5 \mu \mathrm{L}$ of $1 \mathrm{mM}$ methylene blue $\left(\mathrm{C}_{16} \mathrm{H}_{18} \mathrm{~N}_{3} \mathrm{SCl}\right)$ was used for better visualization. The droplet movement by electrowetting was recorded by an optical microscope equipped with a CCD camera. The droplet velocity was obtained by analyzing the recorded video clips (Fig. 5).

Fig. 6 shows the droplet velocity with the fitted curve at various applied voltages; it also shows the theoretically expected contact angle obtained from the Lippmann-Young equation. The droplet velocity exponentially depends on the applied voltage from 45-100 V. The droplet successfully moved back and forth between the ends of the control electrodes at $45 \mathrm{~V}$ and above. The droplet moved to the neighboring electrode with velocities of $0.34 \mathrm{~mm} / \mathrm{s}$ at $45 \mathrm{~V}$ and $32 \mathrm{~mm} / \mathrm{s}$ at $100 \mathrm{~V}$. The threshold voltage $\left(V_{T}\right)$ is the minimum actuation voltage required to move a droplet between two electrodes. The droplet cannot be displaced below the threshold voltage due to the contact angle hysteresis $(\alpha)$ [19]. In short, for droplet transport, the applied 


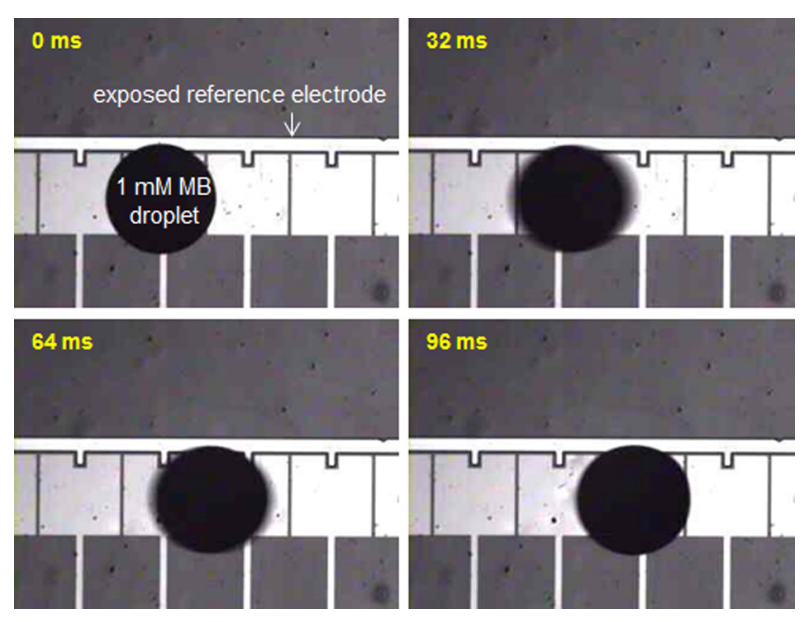

Fig. 5. Time-lapse CCD images of $1 \mathrm{mM}$ methylene blue droplet moved by electrowetting at $90 \mathrm{~V}$.

voltage has to be high enough to decrease the advancing angle to below the inherent receding angle, and overcome adhesive friction related to the contact angle hysteresis. The expected contact angle at $45 \mathrm{~V}$ is $111^{\circ}$, which is the same as the difference between the initial advancing contact angle of $116^{\circ}$ and the contact angle hysteresis of $5^{\circ}$. This result is consistent with the aforementioned theoretical expectation. In case of the unground-type parallel electrode design (Fig. 2(b)), such contact angle change can be theoretically obtained at a two-fold higher voltage. This is because the voltage drop across the dielectric becomes half of the applied voltage. This effect on the threshold voltage for droplet transport related to contact angle hysteresis in various electrowetting configurations is currently under investigation.

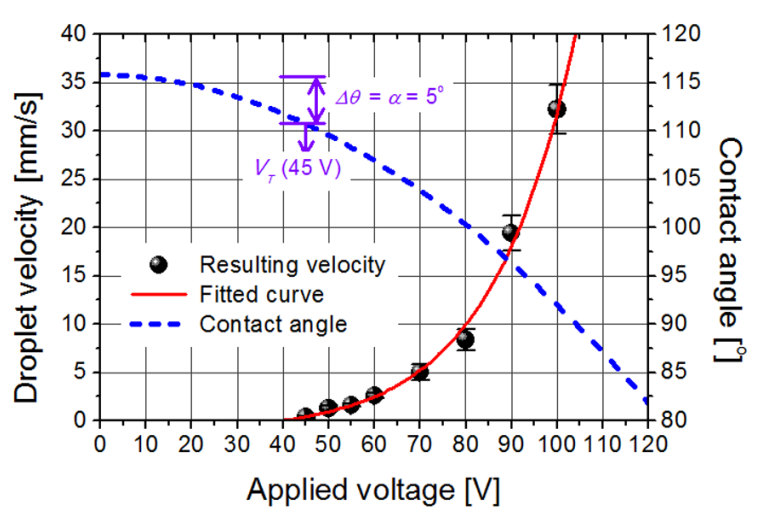

Fig. 6. Measured droplet velocity from the fabricated simplified coplanar ground-type single-plate electrowetting device and theoretically expected contact angle depending on the applied voltage.

These results showed that the proposed method is a simplified fabrication process for a typical coplanar groundtype single-plate electrowetting device. The required voltage for droplet transport by electrowetting can be reduced either by using a high-k thin dielectric or by decreasing the liquid-vapor interfacial tension with surfactants or oil environment [20-23].

In previous ground-type single-plate configurations for droplet transport [Fig. 2(d)-(f)], the grounding reference electrode was formed along the centerline of the control electrode array. Alternately, the reference electrode was railed off from control electrodes to establish contact with the droplet. In such cases, the reference electrode cannot be made on the same plane with the control electrode array. This is because the reference electrode would come into contact with the control electrodes or their wiring lines. On the other hand, in the proposed configuration (Fig. 3), the grounding reference electrode can be made on the same plane with the control electrode array. The single line adjacent to the control electrode array can serve the grounding function without touching the control electrodes or the wiring lines.

The simplified configuration is acceptable within a 2row matrix of the control electrode array, which is similar to other single-plate configurations. For higher dimensional matrix, the electrical wiring for the control electrode array has to be buried under another insulating layer. Interestingly, Paik et al. have reported a similar configuration using two exposed grounding rails by the wayside [24]. However, doing so makes the fabrication process more complex because the reference electrodes require an additional insulating layer for the electrical wiring or throughhole interconnection between control electrode array and contact pads. Actually, one of the reference electrodes is omissible. The nature of the liquid droplet makes it round the contact line and grounded during experiments; therefore, using only a single reference electrode beside the control electrode array results in a simplified fabrication process.

Although the structure of the proposed configuration is transversally asymmetric unlike other configurations, a droplet deviation in the transversal direction is expected to be negligible. Only when the droplet is grounded can the electrowetting force be generated by the activated control electrode underneath the dielectric layer, and not on the reference electrode, which serves the grounding function in direct contact with the droplet.

In electrowetting devices, a transverse groove or protrusion on the substrate surface may cause additional friction against the droplet transport. Therefore, if a groove or protrusion appears in the design, placing these parallel to the direction for droplet motion is recommended.

\section{Conclusion}

A simpler ground-type, single-plate electrowetting device has been successfully fabricated. Its characteristics have been examined to validate the equivalent water droplet transport capability compared with previous similar devices. Although unground-type devices are simpler to 
fabricate, ground-type devices have better electrowetting performance at the same applied voltage. By reducing one photolithography step in the process, the proposed simplified fabrication is possible without degrading the water droplet transport capability. Thus, the process could be simplified as that of unground-type devices. In conclusion, this simplified coplanar ground-type configuration for single-plate electrowetting devices can reduce the number of fabrication steps without any performance degradation.

\section{Acknowledgment}

This work was supported by the Seoul R\&BD Program (No. 10920) and the National Research Foundation of Korea Grant, which is funded by the Korean Government through the Ministry of Education, Science and Technology (Nos. 2009-0075886, K20601000002-09E0100-00200, and NRF-2010-355-D00024).

\section{References}

[1] L. Malic, D. Brassard, T. Veres, and M. Tabrizian, "Integration and detection of biochemical assays in digital microfluidic LOC devices," Lab Chip, vol. 10, no. 4, pp. 418-431, 2010.

[2] M. Abdelgawad and A. R. Wheeler, "The digital revolution: A new paradigm for microfluidics," $A d v$. Mater, vol. 21, no. 8, pp. 920-925, February 2009.

[3] J. Heikenfeld, K. Zhou, E. Kreit, B. Raj, S. Yang, B. Sun, A. Milarcik, L. Clapp, and R. Schwartz, "Electrofluidic displays using Young-Laplace transposition of brilliant pigment dispersions," Nat. Photonics, vol. 3, no. 5, pp. 292-296, May 2009.

[4] R. Shamai, D. Andelman, B. Berge, and R. Hayes, "Water, electricity, and between... On electrowetting and its applications," Soft Matter, vol. 4, no. 1, pp. 38-45, January 2008.

[5] M. G. Pollack, A. D. Shenderov, and R. B. Fair, "Electrowetting-based actuation of droplets for integrated microfluidics," Lab Chip, vol. 2, no. 2, pp. 96-101, May 2002.

[6] M. Abdelgawad, P. Park, and A. R. Wheeler, "Optimization of device geometry in single-plate digital microfluidics," J. Appl. Phys., vol. 105, no. 9, p. 094506, May 2009.

[7] Y. J. Liu, D. J. Yao, H. C. Lin, W. Y. Chang, and H. Y. Chang, "DNA ligation of ultramicro volume using an EWOD microfluidic system with coplanar electrodes," J. Micromech. Microeng., vol. 18, no. 4, 2008.

[8] R. B. Fair, A. Khlystov, T. D. Tailor, V. Ivanov, R. D. Evans, V. Srinivasan, V. K. Pamula, M. G. Pollack, P. B. Griffin, and J. Zhou, "Chemical and biological applications of digital-microfluidic devices," IEEE
Des. Test Comput., vol. 24, no. 1, pp. 10-24, 2007.

[9] Y. Li, M. Yoshio, L. Haworth, W. Parkes, M. Kubota, and A. J. Walton, "Test structure for characterizing low voltage coplanar EWOD system," IEEE Trans. Semicond. Manuf., vol. 22, no. 1, pp. 88-95, 2009.

[10] M. Washizu, "Electrostatic actuation of liquid droplets for micro-reactor applications," IEEE Trans. Ind. Appl., vol. 34, no. 4, pp. 732-737, 1998.

[11] A. Torkkeli, J. Saarilahti, A. Haara, H. Harma, T. Soukka, and P. Tolonen, "Electrostatic transportation of water droplets on superhydrophobic surfaces," in Proceedings of 14th IEEE International Conference on Micro Electro Mechanical Systems, Interlaken, Switzerland, 21-25 January 2001, pp. 475-478.

[12] U.-C. Yi and C.-J. Kim, "Characterization of electrowetting actuation on addressable single-side coplanar electrodes," J. Micromech. Microeng., vol. 16, no. 10, pp. 2053-2059, 2006.

[13] I. Moon and J. Kim, "Using EWOD (electrowettingon-dielectric) actuation in a micro conveyor system," Sens. Actuators A, vol. 130-131, pp. 537-544, 2006.

[14] S.-K. Fan, H. Yang, T.-T. Wang, and W. Hsu, "Asymmetric electrowetting-moving droplets by a square wave," Lab Chip, vol. 7, no. 10, pp. 13301335, 2007.

[15] K. H. Kang, "How electrostatic fields change contact angle in electrowetting," Langmuir, vol. 18, no. 26, p. 10318, 2002.

[16] Y. Fouillet and J.-L. Achard, "Microfluidique discrète et biotechnologie," C. R. Phys., vol. 5, no. 5, pp. 577588, 2004.

[17] R. B. Fair, A. Khlystov, V. Srinivasan, V. K. Pamula, and K. N. Weaver, "Integrated chemical/biochemical sample collection, pre-concentration, and analysis on a digital microfluidic lab-on-a-chip platform," Proc. SPIE, vol. 5591, pp. 113-124, 2004.

[18] C. Cooney, C.-Y. Chen, M. Emerling, A. Nadim, and J. Sterling, "Electrowetting droplet microfluidics on a single planar surface," Microfluid. Nanofluid., vol. 2, no. 5, pp. 435-446, September 2006.

[19] J. Berthier, P. Dubois, P. Clementz, P. Claustre, C. Peponnet, and Y. Fouillet, "Actuation potentials and capillary forces in electrowetting based microsystems," Sens. Actuators A, vol. 134, no. 2, pp. 471-479, March 2007.

[20] H. Moon, S. K. Cho, R. L. Garrell, and C.-J. Kim, "Low voltage electrowetting-on-dielectric," J. Appl. Phys., vol. 92, no. 7, pp. 4080-4087, October 2002.

[21] S. Berry, J. Kedzierski, and B. Abedian, "Low voltage electrowetting using thin fluoroploymer films," J. Colloid Interface Sci., vol. 303, no. 2, pp. 517-524, November 2006.

[22] Y. Li, W. Parkes, L. I. Haworth, A. W. S. Ross, J. T. M. Stevenson, and A. J. Walton, "Room-temperature fabrication of anodic tantalum pentoxide for lowvoltage electrowetting on dielectric (EWOD)," $J$. 
Microelectromech. Syst., vol. 17, no. 6, pp. 14811488, December 2008.

[23] J.-H. Chang, D. Y. Choi, S. Han, and J. J. Pak, "Driving characteristics of the electrowetting-ondielectric device using atomic-layer-deposited aluminum oxide as the dielectric," Microfluid. Nanofluid., vol. 8, no. 2, pp. 269-273, February 2010.

[24] P. Y. Paik, V. K. Pamula, and K. Chakrabarty, "Adaptive cooling of integrated circuits using digital microfluidics," IEEE Trans. Very Large Scale Integr. (VLSI) Syst., vol. 16, no. 4, pp. 432-443, 2008.

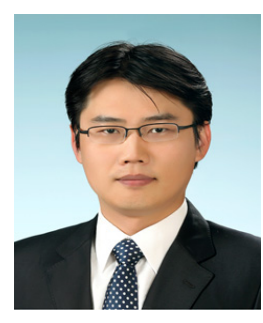

Jong-Hyeon Chang received his B.S., M.S., and Ph.D. degrees in Electrical Engineering in 2003, 2005 and 2010, respectively, from Korea University. $\mathrm{He}$ worked at the Semiconductor Research Institute of Korea University as a research professor. Since 2011, he has been a research staff member of the Material \& Device Research Center at Samsung Advanced Institute of Technology. His research interests include microelectromechanical systems, nano-bioelectronics, and electrowetting-based digital microfluidics.

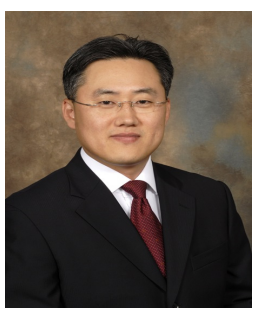

Dong-Sik Kim received his B.S., M.S., and Ph.D. degrees in Medicine in 1996, 1999 and 2004, respectively, from Korea University. He worked at the University of Cincinnati in Ohio, USA from 2006-2009 as a clinical instructor and an assistant professor. Since 2009, he has been working as an assistant professor at the College of Medicine in Korea University. His research interests include biosensor, bio-artificial liver, and hepatocellular carcinoma.

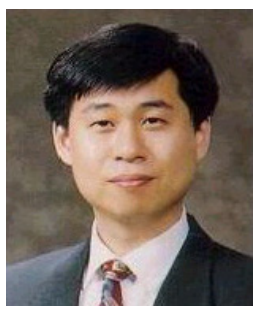

James Jungho Pak received his B.S., M.S., and Ph.D. degrees in Electrical Engineering in 1985, 1988 and 1992, respectively, from Purdue University. $\mathrm{He}$ worked at Intel Corporation in Santa Clara, CA, USA from 1992 1995 as a senior device physicist. Since 1995, he has been a professor at the School of Electrical Engineering in Korea University. His research interests include microsystems (such as bioMEMS), biosensor, applications of polymer in microsensors and actuators, flexible electronics, and novel semiconductor devices and processing. 